\title{
Methylenetetrahydrofolate reductase and psychiatric diseases
}

\author{
Lin Wan', Yuhong Li ${ }^{1}$, Zhengrong Zhang ${ }^{2}$, Zuoli Sun ${ }^{2}, \mathrm{Yi} \mathrm{He}^{2}$ and Rena Li $\mathrm{i}^{1,2,3}$
}

\begin{abstract}
Methylenetetrahydrofolate reductase (MTHFR) is a key enzyme for the critical process of one-carbon metabolism involving folate and homocysteine metabolisms. It is known that some polymorphism of MTHFR would result in reduction of MTHFR enzyme activity as well as DNA methylation process, later shown to have significant impacts in various psychiatric diseases. However, it is unclear whether the polymorphism of MTHFR could be an independent or an add-on risk factor for specific psychiatric symptoms, such as anxiety, depression, positive, or negative symptoms of schizophrenia, or acts as risk factor for specific psychiatric disorders, such as schizophrenia, major depression, autisms, and bipolar disorders. It is also understudied on whether folate supplements could be an effective treatment for psychiatric patients with defect MTHFR activity. In this review, we not only gathered the most recent discoveries on MTHFR polymorphism and related DNA methylation in various psychiatric disorders, but also highlighted the potential relationships between MTHFR activity and implication of folate-related function in specific mental diseases.
\end{abstract}

\section{Introduction}

Methylenetetrahydrofolate reductase (MTHFR) is a key enzyme of folate metabolism in the process of one-carbon metabolism. MTHFR converts 5,10-methylenetetrahydrofolate to 5-methyltetrahydrofolate and participate in folate and homocysteine conversion correlated to DNA methylation $^{1}$. As consequences of polymorphism of MTHFR, reduction of MTHFR enzymatic activity would cause impaired methylation as well as deficiency of folate. There are plenty of relevant studies on linkage between MTHFR and human diseases including cardiovascular diseases, tumors, neurologic diseases, and psychiatric disorders $^{2-5}$. Moreover, there are stratified factors that have been identified to be involved in the relationship between MTHFR and diseases, such as gender, age, and ethnicity $^{6-9}$. As both DNA methylation and folate are important in mental health, reduction of MTHFR activity

\footnotetext{
Correspondence: Rena Li (rli@roskampinstitute.org)

${ }^{1}$ Center for Brain Disorders Research, Capital Medical University \& Beijing

Institute of Brain Disorders, Beijing 100069, China

${ }^{2}$ The National Clinical Research Center for Mental Disorders \& Beijing Key

Laboratory of Mental Disorders of Mental Disorders, Beijing Anding Hospital,

Capital Medical University, Beijing 100088, China
}

Full list of author information is available at the end of the article. or folate deficiency have been associated with an onset of several psychiatric diseases ${ }^{10}$, schizophrenia, bipolar disorder, depression, autism, and ADHD. In this review, we specifically focus on the MTHFR polymorphism and related methylation and folate effects on psychiatric diseases as well as the possibility of relationship between clinical phenotypes of MTHFR-related diseases and effectiveness of clinical treatment in psychiatric patients ${ }^{11}$.

\section{MTHFR \\ MTHFR gene}

In humans, the MTHFR resides on chromosome 1 location p36.3 and was originally described as containing 12 exons as shown in Fig. 1. Human MTHFR transcripts are respectively at $2.2 \mathrm{~kb}, 7.5 \mathrm{~kb}$, and $9.5 \mathrm{~kb}^{12}$. The cDNA of $2.2 \mathrm{~kb}$-fragment sequence codes for a 656 residue and $70-77 \mathrm{kDa} \operatorname{protein}^{13}$. The cDNA of $7.5 \mathrm{~kb}$ and $9.5 \mathrm{~kb}$ sequence code a second isoform of $77 \mathrm{kDa}$ protein. Among the exons of MTHFR, the first one is noncoding ${ }^{1}$. Apart from the coding region, variable 5 ' and 3' noncoding regions (UTR) were identified, resulting in transcript heterogeneity. The 5' and 3' termini of the MTHFR cDNA overlap with the 5 ' terminus of a chloride ion

\section{(c) The Author(s) 2018}

(c) (i) Open Access This article is licensed under a Creative Commons Attribution 4.0 International License, which permits use, sharing, adaptation, distribution and reproduction cc) in any medium or format, as long as you give appropriate credit to the original author(s) and the source, provide a link to the Creative Commons license, and indicate if changes were made. The images or other third party material in this article are included in the article's Creative Commons license, unless indicated otherwise in a credit line to the material. If material is not included in the article's Creative Commons license and your intended use is not permitted by statutory regulation or exceeds the permitted use, you will need to obtain permission directly from the copyright holder. To view a copy of this license, visit http://creativecommons.org/licenses/by/4.0/. 




Fig. 1 MTHFR enzymatic activity with MTHFR mutations. a MTHFR gene exons are indicated as blue regions, and gene $5^{\prime}$ to $3^{\prime}$ end are from right to left. Numbers under the arrows represent the SNP sequence number, which corresponds to the gene rs-number in the annotation. 1. Rs4846080(A > G); 2. rs1476413(G > A); 3. Rs1801131(A > C); 4. rs1801133(C> T); 5. rs17421511(G > A); 6. rs17037396(C > T); 7.rs9651118(T > C); 8. rs17367504(A > G). b Decrease in enzyme Activity (\% of normal) with the presence of MTHFR variants

channel gene and the 3' terminus of an unidentified gene, respectively. The MTHFR gene has multiple promoters and several polyadenylation sites creating 3'UTR lengths of $0.2 \mathrm{~kb} \pm 5.0 \mathrm{~kb}$ or $0.6 \mathrm{~kb} \pm 4.0 \mathrm{~kb}$ in human ${ }^{12}$. The MTHFR gene has been identified to possess 14 common or rare single nucleotide polymorphism that are associated with enzymatic deficiency ${ }^{14}$. Among them rs1801133(C677T) and rs1801131(A1298C) are most reported that may reduce the MTHFR activity in various degrees. For C677T, the enzyme activity of heterozygous and homozygous mutant individuals are respectively 67 and $25 \%$ of the wild-type ones. And for A1298C, the enzyme activity of heterozygous and homozygous mutant individuals are respectively 83 and $61 \%$ of the wild-type subjects $^{15}$, as shown in Fig. 1.

\section{MTHFR and its activity}

While MTHFR gene codes for different variants, the most common form of MTHFR in human is a 656 amino acids protein. Human MTHFR consists of an N-terminal catalytic domain (amino acids 1-356) which binds 5,10methylenetetrahydrofolate (5,10-methylene THF), and a C-terminal regulatory domain (amino acids 363-656) which binds S-adenosylmethionine (AdoMet, SAM) ${ }^{16,17}$. As shown in Fig. 2, MTHFR catalyzes the physiologically irreversible reduction of 5,10-methylene THF to 5methyltetrahydrofolate (5-methyl THF), and plays a critical role in one-carbon metabolism for the reaction of producing methyl groups to participate in epigenetic regulation $^{18}$. The properties and crystal structure of MTHFR from the bacterium Thermus thermophilus HB8 have been determined ${ }^{19}$. While the regulation of MTHFR activity is closely controlled by SAM at C-terminal regulatory domain, more studies indicated that the human MTHFR enzyme activity is also regulated by multiple phosphorylated sites on a serine-rich N-terminal extension region ${ }^{20}$. The phosphorylation leads downregulation of MTHFR activity and upregulation of allosteric inhibition by SAM. It is suggested that phosphorylation impacts on the allosteric regulation of MTHFR via altering the equilibrium of active and inactive states of the enzyme, favoring the inactive state which SAM preferentially binds ${ }^{21}$. The active form of MTHFR could impact on the generation of 5-methyl THF, which is the active form of folate in vivo. Then methionine level increases and related methyl group donation is driven which successively exert potential mechanism on psychiatric diseases, as shown in Fig. 3.

\section{MTHFR and DNA methylation}

Another important role of MTFHR is to participate in donating methyl group to regulate epigenetic modification in the one-carbon metabolism. Methylation is a common regulation process of gene expression that influences cellular development and function ${ }^{22}$, which is dependent on SAM as a methyl donor. SAM originated from methionine cycle in which 5-methyl THF transfers methyl groups to homocysteine in a reaction catalyzed by methionine synthase to produce methionine. In this process, 5,10-methylene THF play a critical role in methionine regeneration and methyl donation, meanwhile MTHFR catalyzes the irreversible conversion of 5,10methylene THF to 5-methyl THF that participate in generation of SAM in methionine cycle and offer methyl group $^{23}$.

\section{MTHFR polymorphism \\ MTHFR polymorphisms and enzymatic activity}

There are several sites of MTHFR polymorphism that have been reported including 2 enzyme activity associated locuses C677T and A1298C and 6 enzyme activity unassociated locuses ${ }^{6}$. As shown in Table 1 , with regard to the 


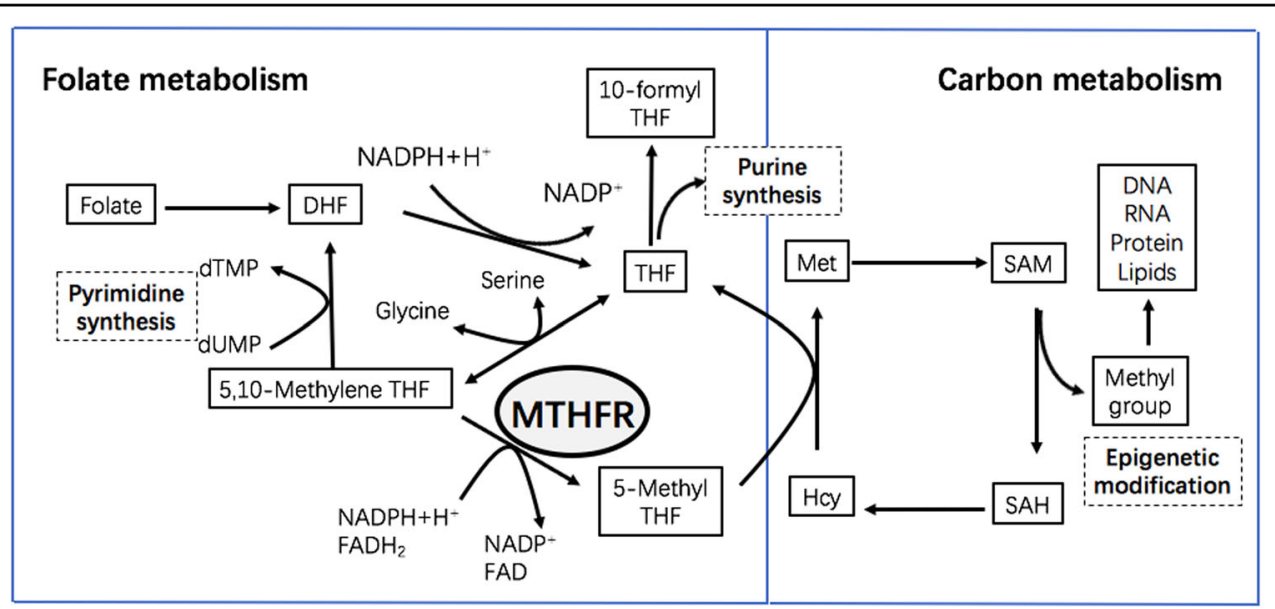

Fig. 2 One-carbon metabolism. MTHFR is a key enzyme to catalyze conversion of 5,10-methylene THF to 5-methyl THF and contribute to generation of SAM, which is the direct donor of methyl group. DHF, dihydrofolate acid; THF, tetrahydrofolate acid; MTHFR, methylenetetrahydrofolate reductase; dTMP, deoxythymidine monophosphate; dUMP, deoxyuridine monophosphate; NADPH, nicotinamide adenine dinucleotide phosphate; FAD, flavine adenine dinucleotide; Met, methionine; Hcy, homocysteine; SAM, S-adenosylmethionine; SAH, S-adenosylhomocysteine

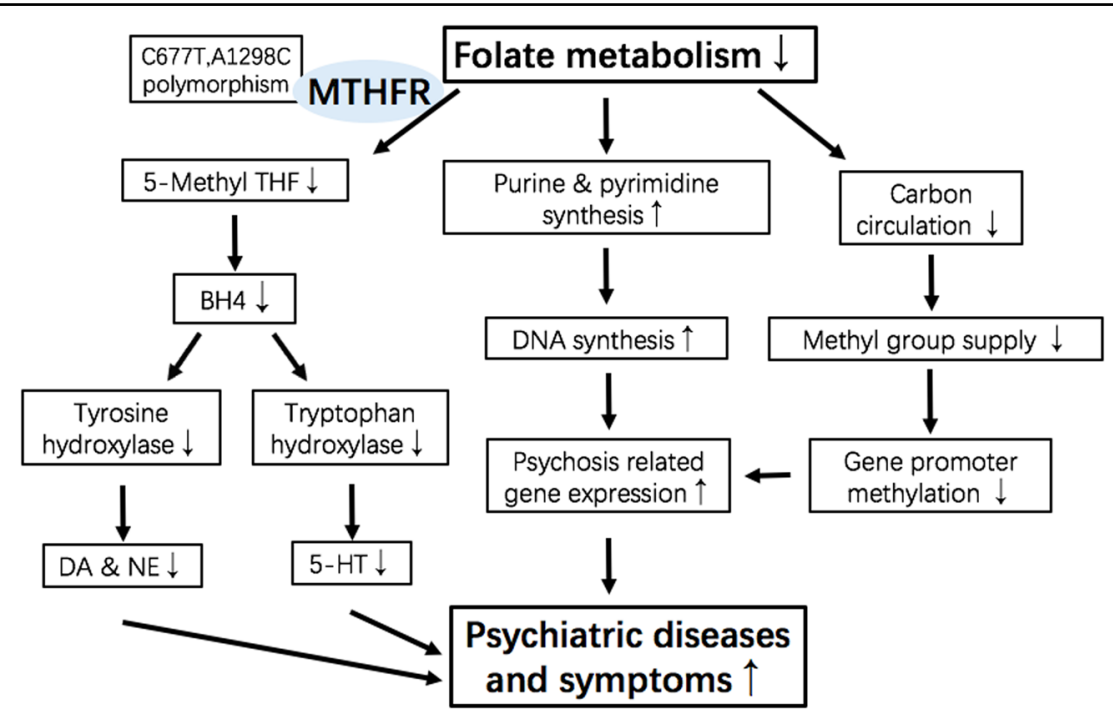

Fig. 3 Potential mechanisms of MTHFR in psychiatric diseases. Methyl group supply in one-carbon metabolism is affected by MTHFR enzyme catalytic process. MTHFR polymorphism affects downstream methylation of schizophrenia-related proteins. DA, glutamate and so on. BH4, tetrahydrobiopterin; DA, dopamine; NE, norepinephrine; 5-HT, 5-hydroxytryptamine

association of MTHFR gene and its enzyme products, some of the studies revealed severe enzymatic deficiency. The encoding of MTHFR appears to be polymorphic such as the gene site C677T, one of the most studied and clinically important variant in exon 4 . The C677T variant results from a single nucleotide substitution at this position, in which cytosine is replaced by thymine resulting a conversion of alanine to valine residue ${ }^{24}$. The substitution lowers the affinity of MTHFR and its cofactor, which promotes the thermolability and diminishes the enzyme activity. Comparing with wild genotype (CC), the heterozygote $(\mathrm{CT})$ and mutation homozygote (TT) lead to the decline of enzyme activity by about 34 and $75 \%$, and increased thermolability in lymphocyte extracts ${ }^{25}$. In 2001, the Ala222Val mutation was created in human MTHFR, and the mutant protein was successfully purified and its properties were determined. Different from the former studies, the Ala222Val variant exhibits identical catalytic properties as the wild-type enzyme, but it is thermolabile ${ }^{17}$.

Another common polymorphism is A1298C, in which adenine is replaced by cytosine resulting a conversion of glutamate to alanine at 429 residue, which also diminishes the enzyme activity. Lymphocyte extracts from 


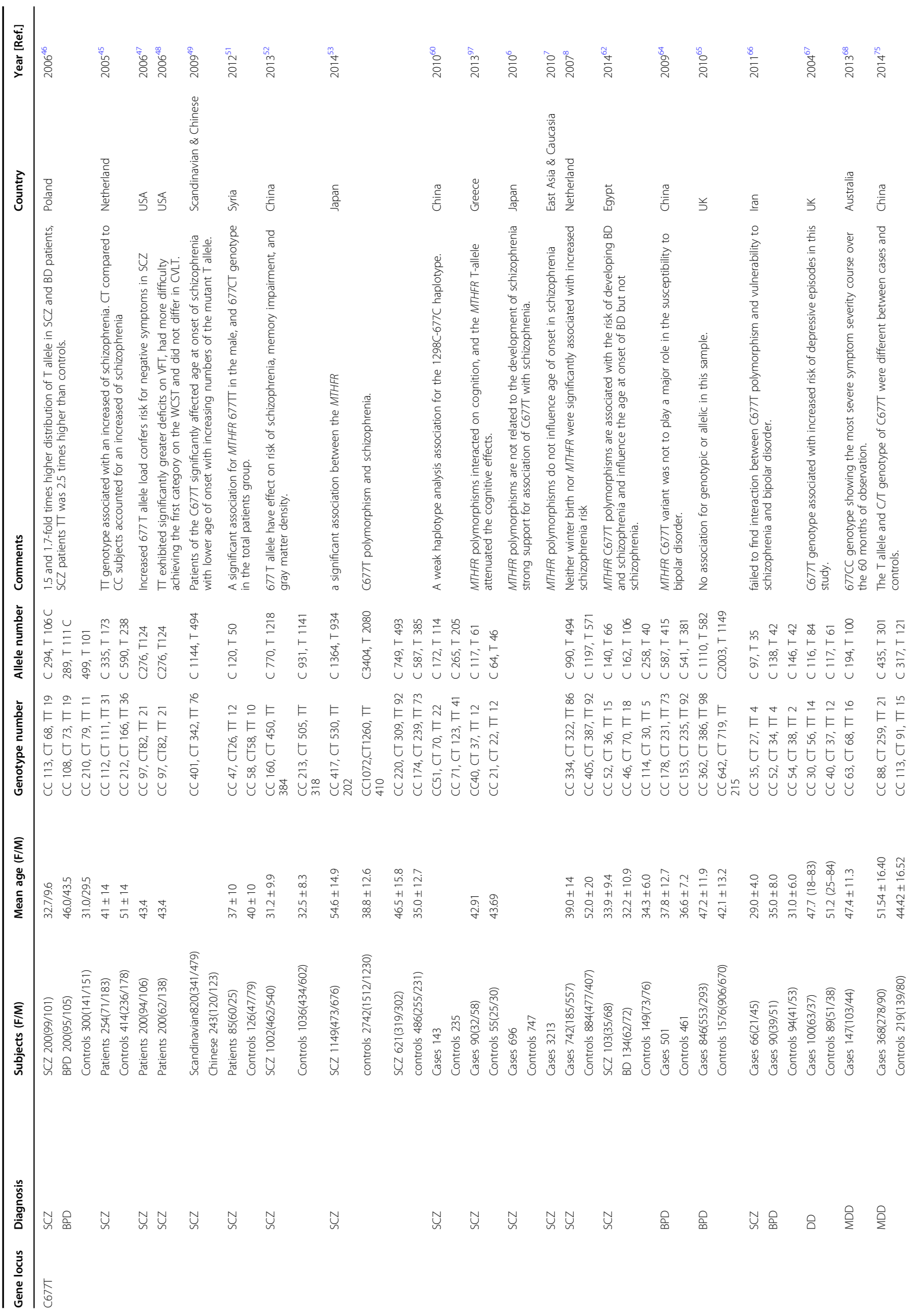







homozygous $1298 \mathrm{CC}$ individuals showed $61 \%$ of wildtype enzyme activity ${ }^{26}$. The Ala177Val was established in the MTHFR of $E$. coli to study the biochemical phenotype of the Ala222Val variant. Then literatures reported the Ala177Val mutation has no influence on the kinetic parameters of bacterial MTHFR, but rather reduces enzyme stability and affinity for cofactor, and thus increases the tendency to form inactive enzyme via flavin dissociation, compared to the wild-type enzyme ${ }^{27}$.

\section{MTHFR polymorphism and methylation}

MTHFR polymorphism is also associated with global methylation activity. For example, a study of coronary artery patients indicated that genomic DNA methylation directly correlates with folate status and inversely with plasma homocysteine levels. After genotype analysis, TT genotypes had a diminished level of global DNA methylation compared with those with CC wild type ${ }^{28}$. Such a change was also found in healthy individuals which showed reduction of DNA methylation in individuals with the TT MTHFR genotype compared to subjects with CC $M T H F R^{29}$. While DNA methylation may be age, gender, and cell-type specific, MTHFR polymorphism might not be always associated with hypomethylation of DNA. For example, a study of aging-related DNA methylation found hypomethylation in aged individuals compared to young populations without significant association with C677T MTHFR genotypes ${ }^{30}$. Studies also demonstrated no significant inference of MTHFR C677T polymorphism in global DNA methylation in oral epithelial cell samples ${ }^{31}$ or lymphocytes of healthy individuals ${ }^{32}$, as well as cutaneous squamous cell carcinoma in renal transplant patients $^{33}$. Those reports suggested a MTHFR polymorphism independent mechanism in aging and cell-type specific global DNA methylation. Furthermore, a similar results were reported in a study of individuals with or without oligozoospermic which showed no significant association between DNA methylation in spermatozoa and the MTHFR C677T genotypes although a trend for higher incidence of methylation alterations in severe oligozoospermic infertile men with CT genotypes were observed $^{34}$, suggesting that a much more complicated or indirect interactions between MTHFR polymorphism and methylation are involved.

As global DNA methylation refers to the average methylation status that occurs across the whole genome, MTHFR polymorphism could also destruct gene-specific methylation process which refers the methylation status of specific genes. For example, a study of MTHFR polymorphism genotypes in colorectal cancer patients reported that the frequency of methylated $\mathrm{Bcl}-2$ promoter was significantly higher in individuals with $\mathrm{CC}$ genotype than that of those with CT and TT genotypes, and a significant difference of $\mathrm{C}$ and $\mathrm{T}$ alleles distribution were observed 
between patients with methylated and unmethylated $\mathrm{Bcl}-2$ promoter $^{35}$. Furthermore, studies of IGF-2 gene in transitional cell carcinoma of the bladder and MGMT gene in gastric cancer showed that patients with CT or TT MTHFR genotypes had reduced methylation of IGF-2 or $M G M T$ compared those with CC genotype ${ }^{36,37}$. Together, as MTHFR is an important enzyme for folate metabolism which plays critical role in epigenetic as DNA methylation, accumulated evidence showed that global DNA methylation can be associated with MTHFR polymorphism genotypes in both healthy populations and individuals with various diseases. However, some cell type- and agerelated global DNA methylation showed independent of MTHFR genotypes. While the underlying mechanism of MTHFR independent global DNA methylation remains unknown, the MTHFR polymorphisms related genespecific DNA methylations were commonly reported in various pathological conditions.

\section{Mouse models of MTHFR deficiency}

The Mthfr of mice were knockout to investigate MTHFR deficient by animal models ${ }^{38}$. The $\mathrm{Mthfr}^{+1-}$ mice showed normal growth and similar survival to that of wild-type mice ${ }^{39}$. The $\mathrm{Mthfr}^{-/-}$mice were with none MTHFR enzyme activity in all tissues, whereas the $\mathrm{Mthfr}^{+/-}$showed $60 \%$ residual activity, similar to the value observed in patients homozygous for the C667T polymorphism $^{40}$. In the $M t h f_{r}{ }^{+-}$and $M t h f_{r}{ }^{-1-}$ mice, the plasma total homocysteine levels were 1.6- and 10-fold higher, respectively, than the wildtype controls. SAM levels were decreased, but S-adenosylhomocysteine (AdoHcy, SAH) levels were elevated considerably, with global DNA hypomethylation observed in both heterozygotes and homozygotes ${ }^{38}$. Then researchers proposed that heterozygous knockout mice appeared to be a good animal model for individuals homozygous for the C667T polymorphism, whereas the homozygous null mice were a better one for severely MTHFR-deficient individuals ${ }^{19}$. Apart from human studies, mice with heterozygous and homozygous mutation in Mthfr C677T still accompany with global DNA hypomethylation, decreased SAM and increased SAH levels ${ }^{41}$.

\section{MTHFR polymorphism and psychiatric diseases}

Extensive clinical studies demonstrated a significant linkage between MTHFR polymorphism and various diseases, such as cardiovascular diseases, neuronal developmental diseases, cancers as well as psychiatric disorders. Among which, C677T and A1298C polymorphisms of MTHFR have been studied the most in psychiatric diseases and showed significant association with reduction of MTHFR enzymatic activity and methylation. In this session, we will focus on the polymorphisms in the gene encoding for MTHFR in schizophrenia (SZ), bipolar disorder (BPD), depression, autism disorder (ASD) and attention deficit hyperactivity disorder (ADHD). Table 1. summarizes studies including MTHFR polymorphism and psychiatric diseases involved in this review.

\section{Schizophrenia}

For decades ago, there was a report of MTHFR enzymatic activity reduction in two schizophrenia patients which were 18 and $21 \%$ percent of the normal level, respectively, while homocysteine remethylation was also defected $^{42}$. Later, a regression model was created in a study of MTHFR C677T genotype and DNA methylation in schizophrenia subjects, which found females with TT genotype were associated with the lowest global methylation $^{43}$.

Amounts of studies have demonstrated that the level of MTHFR polymorphism in C677T locus is associated with the risk of schizophrenia. As indicated in a meta-analysis of MTHFR consisted of 7 studies, individuals carried with TT homozygotes had the greatest risk of schizophrenia, compared to the subjects with CC wild type and CT heterozygous genotypes ${ }^{44}$. An allele study with welldefined patients and healthy controls indicated that people with CT heterozygotes had the higher risk of schizophrenia than CC carriers ${ }^{45}$. Furthermore, a genotype study also reported that homozygous TT genotype of MTHFR was also associated with risk of schizophrenic patients accompanying with bipolar disorder ${ }^{46}$.

It is interesting to mention that the C677T polymorphisms of MTHFR also has an influence on symptoms of schizophrenia. For example, an increased $\mathrm{T}$ allele load is linked to the increase severity of negative symptoms in schizophrenia, while reducing severity of positive symptoms were also noticed. However, the effect of $\mathrm{T}$ allele on the negative symptoms of schizophrenia could be further enhanced by folate deficiency ${ }^{47}$. Furthermore, comparing with CC and CT, schizophrenia patients with TT genotype exhibited greater deficits on the verbal fluency test (VFT) and more difficulties on the Wisconsin Card Sorting Test (WCST), but not in California Verbal Learning Test (CVLT) performance ${ }^{48}$. However, the effect of C677T polymorphisms of MTHFR on cognitive function was not significant in normal subjects as a longitudinal cognitive study showed that the MTHFR C677T polymorphism was not associated with cognitive performance at baseline or over 12 years ${ }^{49}$. In addition, studies also demonstrated that the C677T polymorphism of MTHFR is associated with onset age of schizophrenia in a dose-dependent manner, such as increasing numbers of the mutant $\mathrm{T}$ allele is linked with early onset ${ }^{50}$.

The relationship between MTHFR polymorphism and schizophrenia in different ethnic population were also investigated. Study of schizophrenic patients and healthy controls in the Arab population from Syria found a strong 
association between C677T and schizophrenia, which showed higher variant $\mathrm{T}$ allele frequency in the patients group. Interestingly, a statistically significant association was found for 677TT genotype under the recessive model in the male patients subgroup, and CT genotype under the overdominant model in the total patients group ${ }^{51}$. Studies of Chinese Han population indicated that the $\mathrm{T}$ allele shown associated with schizophrenia as a risk allele $^{52}$ while a case-control association between the MTHFR C677T polymorphism and schizophrenia in a Japanese subjects research also demonstrated a strong linkage between the MTHFR C677T polymorphism and schizophrenia ${ }^{53}$. Furthermore, a meta-analysis including 38 studies with schizophrenia cases and controls showed the association between $\mathrm{C} 677 \mathrm{~T}$ polymorphism and risk of schizophrenia in all three ethnic populations-African, Asian, and Caucasian ${ }^{54}$.

Studies of sex differences in MTHFR polymorphism might provide some insights for the divergent results from various studies of psychiatric disorders. A strong association between $677 \mathrm{~T}$ allele and male patients with schizophrenia compared female patients suggest that 677T allele might represent different liability in genders ${ }^{46}$. While little is known on the sex differences in MTHFR polymorphisms, sex hormones, such as estrogen is known to play a protective effect in female patients with schizophrenia as for the impact of neurodevelopment and social maturation ${ }^{55}$. On the other hand, testosterone may increase male vulnerability to an adverse illness course compared to estrogen ${ }^{56}$, attributed to its narrower and sometimes unfavorable neuroprotection and neurotransmitter modulation profile ${ }^{57}$. Furthermore, progesterone is reported to benefit neurocognition though enhancement of dopamine release in human males and may also have relevance in male physical and mental health while enhancing the benefits of estrogen through potentiation of estrogen-primed effects on dopamine receptors in male schizophrenic patients ${ }^{58}$.

Except for the C677T, there is another site of MTHFR polymorphisms associated with psychiatric disorders. A study with patients of schizophrenia and control subjects showed an association between the A1298C allele and schizophrenia ${ }^{59}$. Another research including 111 families, demonstrated that deficient MTHFR enzyme activity in pregnant women was related to the A1298C variant, which was associated with a higher risk of schizophrenia in the offsprings ${ }^{60}$.

Studies of individual with both SNPs (C677T and A1298C) showed that subjects with heterozygosity for both mutations resulted in an even lower MTHFR activity than heterozygosity for single MTHFR mutations, while no subjects carry both homozygote for MTHFR mutations regardless which SNPs ${ }^{15}$ Furthermore, There were studies of multiple polymorphisms of one-carbon metabolism and schizophrenia symptoms showed an increase negative symptoms severity with increase of risk alleles, suggesting a cumulative effects of risk SNPs in one-carbon metabolism $^{61}$.

\section{Bipolar disorder}

In addition to schizophrenia, study demonstrated an association between homozygous 677TT genotype of MTHFR gene and bipolar disorder with stronger linkage in male patients than female patients ${ }^{46}$. Another study found a higher prevalence of C677T polymorphism in BD patients than healthy subjects, while patients with $\mathrm{BD}$ with early onset carried one copy of the $\mathrm{T}$ allele ${ }^{62}$. A meta-analysis of 56 studies examining MTHFR C677T in patients and control subjects indicated that the $\mathrm{T}$ allele and TT genotype carriers showed significant increased risk of major psychiatric disorders including schizophrenia and bipolar disorder ${ }^{63}$. At the same time, some studies found disparate results. For instance, a study reported no significant association between C677T and bipolar disorder ${ }^{64}$, while another study found no evidence for C677T genotypic or allelic association with BD regardless of type $\mathrm{I}$ or $\mathrm{II}^{65}$. A study with bipolar patients and schizophrenia subjects also observed no robust differences between patients and controls either for allele frequencies or genotype distribution of C677T polymorphism ${ }^{66}$. These discrepancies may result from population stratifications, explicitly, socio-economic status. On the other hand, the included sample size may play a critical role in divergent results.

\section{Depression}

Depression is another major psychiatric disease. MTHFR polymorphism is also noticed in patients with depression. Studies found that MTHFR polymorphisms might be related to the episode and prognosis of depressive disorder, not the stage of the disease. For example,a cohort study of depressive patients and healthy controls found that MTHFR polymorphism were more common in the individuals with depression history compared to controls ${ }^{67}$, while a study over a 60-month follow-up with depressed subjects indicated that the CC genotype of MTHFR C677T were more likely to have more severe symptoms compared to TT genotype carriers ${ }^{68}$. Another study showed that hyperhomocysteinemia and TT MTHFR genotype were significantly related to depression only, not comorbid anxiety disorder ${ }^{69}$. More studies reported that MTHFR C677T is associated with risk of depression, such as postmenopausal depression ${ }^{70}$ and childhood trauma related major depression disorder $(\mathrm{MDD})^{71}$. It is important to point out the interaction between MTHFR polymorphisms and environmental risks for MDD, such as dietary and stress. For example, a study of inter-relationship between MTHFR polymorphism and 
MDD found that the minor T-allele of MTHFR C677T was associated with increased folate deficiency-related body mass index and homocysteine levels in MDD patients only ${ }^{72}$. Another stress-related MTHFR polymorphism in MDD study showed that traumatic stress in childhood could increase risk of MDD recurrence as well as the development of more severe depressive symptoms in MTHFR TT genotype carriers. This study suggests that the increase of mutant allele number of $\mathrm{T}$ in C677T locus will enhance stress risk for depression ${ }^{71}$. Both above studies suggest that MTHFR polymorphisms might enhance the environmental risks (low folate intake, traumatic stress at childhood) for MDD via the interaction between genetic and environmental factors. Such a risk was confirmed by a meta-analysis recruiting 26 published studies which showed an association between MTHFR C677T polymorphism and increased risk of depression ${ }^{73}$. However, some studies showed no association between MTHFR and MDD or antidepressant treatment response ${ }^{74,75}$.

Similarly, diverse situation existed in other researches as a study did not find evidence of an association between the MTHFR TT genotype and depression in a depression cohort $^{76}$. Another study including depressed subjects indicated no significant differences in frequency of the $\mathrm{T}$ allele or TT genotype between the depressed and healthy controls $^{77}$. A research of TT genotype and depression scores revealed that the $\mathrm{C} 677 \mathrm{~T}$ gene variation does not play an important role in the depression scores ${ }^{78}$. In a meta-analysis, no significant differences in genotype or allele frequencies between depressive patients and controls were observed ${ }^{74}$.

A possible reason for divergent consequences is population stratification as the frequency of the $\mathrm{T}$ allele is subject to considerable ethnic and geographic variation ${ }^{74}$. Another possibility is that there is an association of this SNP with another disease that is highly correlated with depression. Indeed it has been hypothesized that depression and vascular disease may be different manifestations of the same genetic substrates ${ }^{79}$. Both of these conditions are a result of the interaction of multiple genetic factors and environment, involving multiple genes with small interactive and additive effects.

\section{Autism disorder}

Comparing to Schizophrenia and depression, relatively limited studies of MTHFR in autism have been conducted. Some studies showed higher frequency of C677T polymorphism in children with ASD than in healthy controls $^{80}$, or associated with ASD behavior phenotypes ${ }^{81}$. A risk study of ASD with typical development indicated significant interaction effects between maternal TT genotype and greater risk for $\mathrm{ASD}^{82}$, suggesting MTHFR polymorphism might involve the early development of ASD. Other studies in the Chinese Han and Korean population also found that MTHFR C677T and A1298C mutation genes were risk factors for autism in Chinese Han children and Korean population, respectively ${ }^{83,84}$.

\section{Attention deficit hyperactivity disorder (ADHD)}

In terms of the relationship between MTHFR and ADHA, only very few studies have been reported, even with controversial findings. For example, studies demonstrated that A1298C genotype appeared to be the predominant linkage to the inattentive symptoms, leading to a 7.4-fold increase in ADHD, compared with a 1.3-fold increase for the C677T genotype ${ }^{85}$, individuals with ADHD seem to be related to A1298C polymorphisms ${ }^{86}$. However, a research with ADHD and healthy controls reported no association between C677T or A1298C polymorphism and ADHD in Turkish children ${ }^{87}$. Further studies with large sample size or better controls are needed.

In conclusion, $M T H F R$ polymorphism not only increase risks for diabetes, cardiovascular diseases, and various cancers, but also increase the risk for various psychiatric diseases. For example, as we described above that MTHFR polymorphism is associated with early onset of schizophrenia and the severity of depressive symptoms in MDD. This is important since neurotransmitter imbalances hypotheses are still the main streams for schizophrenia and MDD. Understanding alternative mechanisms of psychiatric diseases will not only provide potential biomarkers for specific psychiatric diseases, but also new targets for antipsychotic drug development. Due to significant controversial findings in MTHFR mutation and DNA methylation in both healthy populations and psychiatric patients, investigation of MTHFR activity in peripheral samples might be important. As yet, the relationships between enzymatic activity and mutation of MTHFR have been reported in general healthy and mental retardation populations as well as in animals, no studies have been found in clinical test of MTHFR activity in psychiatric patients ${ }^{88-90}$. In addition, there are still some shortages on MTHFR mutation and psychiatric disease studies. Except for C677T and A1298C, there were little studies on other SNPs as well as the effect of multiple SNPs on the diseases which may also affect MTHFR activity.

\section{Clinical treatment strategy for MTHFR-related psychiatric disorders}

As MTHFR plays a critical role in one-carbon metabolism, which is composed of folate, homocysteine, vitamin B12, and methylation of DNA, mutation of specific gene locus on MTHFR and correlative enzyme activity decline will affect various of physiological events as well as some pathology states, including psychiatric disorders. Whether we could cope with gene mutation and enzyme 
activity damage using folate one-carbon metabolism strategy as clinical treatment for MTHFR-related psychiatric disease? Some studies showed some interesting possibilities. For example, studies of healthy females found that the low level of serum folate in 677TT genotype is associated with an increase in homocysteine concentration and DNA hypomethylation ${ }^{91,92}$, which reveals the association between MTHFR C677T polymorphisms and nutrient status. As food is a major resource for folate, studies reported that low folate level due to unbalanced diet is associated with higher prevalence on schizophrenia, particularly in infants with maternal nutritional deficiency ${ }^{11,93}$. Another study exploring the association between folate and symptoms of schizophrenia indicated that low folate was associated with negative symptoms severity in schizophrenia subjects ${ }^{94}$. One possible role of folate in mental health is its action on DNA methylation and gene expression which have been wildly reported in human psychiatric disorders.

As MTHFR polymorphisms-induced MTHFR activity decline is irreversible, clinicians tried to use supplement of folate to help methylation process and change the pathogenesis state. For instance, methylfolate supplement was used for the improvement of psychiatric symptoms ${ }^{95}$, while folate supplementation showed reduction of the incidence of neural tube defects which reduces the incidence of schizophrenia ${ }^{96}$. Although there is no evidence that supplements are helpful in the treatment of psychosis in general, based on the published studies, we believe that if we can detect MTHFR polymorphism in individuals with various psychiatric diseases, we might be able to differentiate those MTHFR-related psychiatric patients from non MTHFR deficient patients and develop specific clinical treatment strategies, such as folate or methylfolate supplement to reverse the symptoms. In summary, due to the higher frequency of MTHFR polymorphism in various psychiatric disease, supplement of folate and cobalamin might be critical when patients with MTHFR deficiency. MTHFR deficiency-related psychiatric diseases should be identified and might be able to be treated with targeted supplement for the diseases and related symptoms.

\section{Conclusions}

Increasing evidence demonstrated that MTHFR polymorphism including C677T and A1298C is associated with psychiatric diseases. The MTHFR gene polymorphism is linked to onset, clinical symptoms, prevalence as well as response to treatments. The influence of MTHFR on psychiatric diseases is mainly through reduction of MTHFR activity which results in elevation of homocysteine, reduction of DNA methylation-dependent methyl donor, finally induces hypomethylation, and then active disease-related genes. However, some age- and cell type-specific methylation seems independent from MTHFR polymorphism. MTHFR mutation also can increase environmental risks for psychiatric disorders, such as MDD through interaction between genetic and epigenetic factors. Investigation of MTHFR in psychiatric diseases has important clinical implications, such as identification role of MTHFR and its genotypes in the psychiatric patients who respond or not respond to traditional pharmacological treatment for personalized treatment management of psychiatric diseases.

\section{Acknowledgements}

We thank all researchers and colleagues from Key Laboratory of Molecular Biology in Beijing Anding Hospital of Capital Medical University and Beijing Institute for Brain Disorders for their great support

\section{Author details}

${ }^{1}$ Center for Brain Disorders Research, Capital Medical University \& Beijing Institute of Brain Disorders, Beijing 100069, China. ${ }^{2}$ The National Clinical Research Center for Mental Disorders \& Beijing Key Laboratory of Mental Disorders of Mental Disorders, Beijing Anding Hospital, Capital Medical University, Beijing 100088, China. ${ }^{3}$ Center for Hormone Advanced Science and Education, Roskamp Institute, Sarasota, FL 34243, USA

\section{Competing interests}

The authors declare that they have no conflict of interest.

\section{Publisher's note}

Springer Nature remains neutral with regard to jurisdictional claims in published maps and institutional affiliations.

Received: 24 January 2018 Revised: 7 August 2018 Accepted: 10 September 2018

Published online: 05 November 2018

\section{References}

1. Froese, D. S. et al. Mutation update and review of severe methylenetetrahydrofolate reductase deficiency. Hum. Mutat. 37, 427-438 (2016).

2. Oztuzcu, S. et al. Evaluation of Factor V G1691A, prothrombin G20210A, Factor XIII V34L, MTHFR A1298C, MTHFR C677T and PAI-1 4G/5G genotype frequencies of patients subjected to cardiovascular disease (CVD) panel in south-east region of Turkey. Mol. Biol. Rep. 41, 3671-3676 (2014).

3. Ferrara, M., Capozzi, L. \& Russo, R. Impact of the MTHFR C677T polymorphism on risk of Wilms tumor: case-control study. J. Pediatr. Hematol. Oncol. 31, 256-258 (2009).

4. Mansoori, N., Tripathi, M., Luthra, K., Alam, R., Lakshmy, R. \& Sharma, S. et al. MTHFR (677 and 1298) and IL-6-174 G/C genes in pathogenesis of Alzheimer's and vascular dementia and their epistatic interaction. Neurobiology of Aging 33, 1003.e1-1003.e8 (2012).

5. An, X. K. et al. Association of MTHFR C677T polymorphism with susceptibility to migraine in the Chinese population. Neurosci. Lett. 549, 78-81 (2013).

6. Yoshimi, A. et al. Gene-wide association study between the methylenetetrahydrofolate reductase gene (MTHFR) and schizophrenia in the Japanese population, with an updated meta-analysis on currently available data. Schizophr. Res 124, 216-222 (2010).

7. Saetre, P. et al. Methylenetetrahydrofolate reductase (MTHFR) C677T and A1298C polymorphisms and age of onset in schizophrenia: a combined analysis of independent samples. Am. J. Med Genet B Neuropsychiatr. Genet 156, 215-224 (2011).

8. Muntjewerff, J. W. et al. Effects of season of birth and a common MTHFR gene variant on the risk of schizophrenia. Eur. Neuropsychopharmacol. 21, 300-305 (2011).

9. Muntjewerff, J. W. et al. No evidence for a preferential transmission of the methylenetetrahydrofolate reductase $677 \mathrm{~T}$ allele in families with 
schizophrenia offspring. Am. J. Med Genet B Neuropsychiatr. Genet 144B, 891-894 (2007).

10. Klengel, T., Pape, J., Binder, E. B. \& Mehta, D. The role of DNA methylation in stress-related psychiatric disorders. Neuropharmacology 80, 115-132 (2014).

11. St Clair, D. et al. Rates of adult schizophrenia following prenatal exposure to the Chinese famine of 1959-1961. JAMA 294, 557-562 (2005).

12. Tran, P. et al. Multiple transcription start sites and alternative splicing in the methylenetetrahydrofolate reductase gene result in two enzyme isoforms. Mamm. Genome 13, 483-492 (2002).

13. Goyette, P. P. A. \& Milos, R. et al. Gene structure of human and mouse methylenetetrahydrofolate reductase (MTHFR). Mamm. Genome 9, 652-656 (1998).

14. Liew, S. C. \& Gupta, E. D. Methylenetetrahydrofolate reductase (MTHFR) C677T polymorphism: epidemiology, metabolism and the associated diseases. Eur. J. Med Genet 58, 1-10 (2015).

15. van der Put, N. M. et al. A second common mutation in the methylenetetrahydrofolate reductase gene: an additional risk factor for neural-tube defects? Am. J. Hum. Genet 62, 1044-1051 (1998).

16. Robert Pejchal, EC. et al. Structural perturbations in the Ala-Val polymorphism of methylenetetrahydrofolate reductase-how binding of folates may protect against inactivation. Biochemistry 45, 4808-4818 (2006).

17. Kazuhiro Yamada, Z. C., Rima, R. \& Rowena Mathews, G. Effects of common polymorphisms on the properties of recombinant human methylenetetrahydrofolate reductase. Proc. Natl Acad. Sci. USA 98, 14853-14858 (2001).

18. Selhub, PJBJ. A common mutation in the methylenetetrahydrofolate reductase gene is associated with an accumulation of formylated tetrahydrofolates in red blood cells. Proc Natl Acad Sci USA 95, 13217-13220 (1998).

19. Trimmer, E. Methylenetetrahydrofolate reductase: biochemical characterization and medical significance. Curr. Pharm. Des. 19, 2574-2593 (2013).

20. Yamada, K., Strahler, J. R., Andrews, P. C. \& Matthews, R. G. Regulation of human methylenetetrahydrofolate reductase by phosphorylation. Proc. Natl Acad. Sci. USA 102, 10454-10459 (2005).

21. G.Matthews, D. A. Ja Allosteric inhibition of methylenetetrahydrofolate reductase by adenosylmethionine. J. Biol. Chem. 262, 2485-2493 (1987).

22. Krebs, M. O., Bellon, A., Mainguy, G., Jay, T. M. \& Frieling, H. One-carbon metabolism and schizophrenia: current challenges and future directions. Trends Mol. Med 15, 562-570 (2009).

23. de Arruda, I. T., Persuhn, D. C. \& de Oliveira, N. F. The MTHFR C677T polymorphism and global DNA methylation in oral epithelial cells. Genet Mol. Biol. 36, 490-493 (2013)

24. Frosst, P. B. H. et al. A candidate genetic risk factor for vascular disease-a common mutation in methylenetetrahydrafolate reductase. Nat. Genet 1994 111-113 (1995).

25. PhilippeGoyette, B., . \& David S, R. \& Rozen, R. Severe and mild mutations in cis for the Methylenetetrahydrofolate Reductase (MTHFR) gene, and description of five novel mutations in MTHFR. Am. J. Human. Genet. 59, 1268-1275 (1996).

26. Ilan Weisberg, P. T., Benedicte, C., Sahar, S. \& Rima Rozen, A. Second genetic polymorphism in Methylenetetrahydrofolate Reductase (MTHFR) associated with decreased enzyme activity. Mol. Genet. Metab. 64, 169-172 (1998).

27. Brian, D. et al. The structure and properties of methylenetetrahydrofolate reductase from Escherichia coli suggest how folate ameliorates human hyperhomocysteinemia. Nat. Struct. Biol. 6, 359 (1999).

28. Friso S, C. S. W. et al. A common mutation in the 5,10-methylenetetrahydrofolate reductase gene affects genomic DNA methylation through an interaction with folate status. Proc. Natl Acad. Sci. USA 99, 5606-5611 (2002).

29. Choi, L. S. J. M. J. S. S. Genomic DNA hypomethylation, a characteristic of most cancers, is present in peripheral leukocytes of individuals who are homozygous for the C677T polymorphism in the methylenetetrahydrofolate reductase gene. Cancer Epidemiol. Biomark. Prev. 9, 849 (2000)

30. Marcus, V. M. \& et al. Age-related changes in the global DNA methylation profile of leukocytes are linked to nutrition but are not associated with the MTHFR C677T genotype or to functional capacities. Plos ONE 7, e52570 (2012).

31. Isabela Tatiana Sales de Arruda, D. C. Pa. N. F. Pd. O. The MTHFR C677T polymorphism and global DNA methylation in oral epithelial cells. Genet. Mol. Biol. 36, 490-493 (2013).

32. Narayanan, S. et al. Associations between two common variants C677T and A1298C in the methylenetetrahydrofolate reductase gene and measures of folate metabolism and DNA stability (strand breaks, misincorporated uracil, and DNA methylation status) in human lymphocytes in vivo. Cancer Epidemiol. Biomark. Prev. 13, 1436-1443 (2004).

33. Hanks, J. et al. The association between MTHFR $677 \mathrm{C}>\mathrm{T}$ genotype and folate status and genomic and gene-specific DNA methylation in the colon of individuals without colorectal neoplasia. Am. J. Clin. Nutr. 98, 1564-1574 (2013).

34. Louie, $\mathrm{K}$. et al. Evaluation of DNA methylation at imprinted DMRs in the spermatozoa of oligozoospermic men in association with MTHFR C677T genotype. Andrology 4, 825-831 (2016).

35. Zhu, Q. et al. Impact of MTHFR gene C677T polymorphism on Bcl-2 gene methylation and protein expression in colorectal cancer. Scand. J. Gastroenterol. 46, 436-445 (2011).

36. Huan Cheng, Z. D., Zengjun, W., Wei, Z. \& Jiantang, S. MTHFR C677T polymorphisms are associated with aberrant methylation of the IGF-2 gene in transitional cell carcinoma of the bladder. J. Biomed. Res. 26, 77-83 (2012).

37. Xia, Y., . \& Song, B. \& Kong, X. \& Liu, D. \& Li, J. Aberrant DNA Methylation of P16, MGMT, and hMLH1 Genes in Combination with MTHFR C677T Genetic Polymorphism in gastric cancer. Pak J Med Sci 29, 1338-1343 (2013).

38. Lawrance, A. K. et al. Complete deficiency of methylenetetrahydrofolate reductase in mice is associated with impaired retinal function and variable mortality, hematological profiles, and reproductive outcomes. J. Inherit. Metab. Dis. 34, 147-157 (2011).

39. Schwahn, B. C. et al. Betaine rescue of an animal model with methylenetetrahydrofolate reductase deficiency. Biochem J. 382, 831-840 (2004).

40. Christensen, B. et al. Correlation of a common mutation in the methylenetetrahydrofolate reductase gene with plasma homocysteine in patients with premature coronary artery disease. Arter. Throm Vas 17, 569-573 (1997).

41. Chen Z, K. A. C. et al. Mice deficient in methylenetetrahydrofolate reductase exhibit hyperhomocysteinemia and decreased methylation capacity, with neuropathology and aortic lipid deposition. Human. Mol. Genet. 10, 433 (2001).

42. Freeman, J. M., Finkelstei, J. D. \& Mudd, S. H. Folate-responsive homocystinuria and "schizophrenia". Nutr. Rev. 40, 242-245 (2010).

43. Burghardt, K. J., Pilsner, J. R., Bly, M. J. \& Ellingrod, V. L. DNA methylation in schizophrenia subjects: gender and MTHFR 677C/T genotype differences. Epigenomics 4, 261-268 (2012).

44. Lewis, S. J., Zammit, S., Gunnell, D. \& Smith, G. D. A meta-analysis of the MTHFR C677T polymorphism and schizophrenia risk. Am. J. Med Genet B Neuropsychiatr. Genet 135B, 2-4 (2005).

45. Muntjewerff, J. W. et al. Hyperhomocysteinemia, methylenetetrahydrofolate reductase 677TT genotype, and the risk for schizophrenia: a Dutch population based case-control study. Am. J. Med Genet B Neuropsychiatr. Genet 135B, 69-72 (2005).

46. Kempisty, B. et al. Association of 677C>T polymorphism of methylenetetrahydrofolate reductase (MTHFR) gene with bipolar disorder and schizophrenia. Neurosci. Lett. 400, 267-271 (2006).

47. Roffman, J. L. et al. Contribution of methylenetetrahydrofolate reductase (MTHFR) polymorphisms to negative symptoms in schizophrenia. Biol. Psychiatry 63, 42-48 (2008).

48. Roffman, J. L. et al. Effects of the methylenetetrahydrofolate reductase (MTHFR) C677T polymorphism on executive function in schizophrenia. Schizophr. Res. 92, 181-188 (2007).

49. Schiepers O J, vB. M. P. et al. Genetic variation in folate metabolism is not associated with cognitive functioning or mood in healthy adults. Progress. Neuro-Psychopharmacol. Biol. Psychiatry 35, 1682-1688 (2011).

50. Vares, M. et al. Association between methylenetetrahydrofolate reductase (MTHFR) C677T polymorphism and age of onset in schizophrenia. Am. J. Med Genet B Neuropsychiatr. Genet 153B, 610-618 (2010).

51. Lajin, B., Alhaj Sakur, A., Michati, R. \& Alachkar, A. Association between MTHFR C677T and A1298C, and MTRR A66G polymorphisms and susceptibility to schizophrenia in a Syrian study cohort. Asian J. Psychiatr. 5, 144-149 (2012)

52. Zhang, Y. et al. Association of MTHFR C677T polymorphism with schizophrenia and its effect on episodic memory and gray matter density in patients. Behav. Brain Res 243, 146-152 (2013).

53. Nishi, A. et al. Meta-analyses of blood homocysteine levels for gender and genetic association studies of the MTHFR C677T polymorphism in schizophrenia. Schizophr. Bull. 40, 1154-1163 (2014).

54. Yadav, U., Kumar, P., Gupta, S. \& Rai, V. Role of MTHFR C677T gene polymorphism in the susceptibility of schizophrenia: an updated meta-analysis. Asian J. Psychiatry 20, 41-51 (2016). 
55. da Silva, T. L. \& Ravindran, A. V. Contribution of sex hormones to gender differences in schizophrenia: a review. Asian J. Psychiatr. 18, 2-14 (2015).

56. Salokangas, R. K. R. Gender and the use of neuroleptics in schizophrenia. Schizophr. Res. 66, 41-49 (2004).

57. Ebinger, M., Sievers, C., Ivan, D., Schneider, H. J. \& Stalla, G. K. Is there a neuroendocrinological rationale for testosterone as a therapeutic option in depression? J. Psychopharmacol. 23, 841-853 (2009).

58. Lee, D. et al. Progesterone modulation of D5 receptor expression in hypothalamic ANP neurons, the role of estrogen. Mol. Psychiatry 6, 112-117 (2001).

59. Zhang, C. et al. Further evidence that methylenetetrahydrofolate reductase A1298C polymorphism is a risk factor for schizophrenia. J. Neural Transm. Nienna) 117, 1115-1117 (2010).

60. Zhang, C. et al. Influence of maternal MTHFR A1298C polymorphism on the risk in offspring of schizophrenia. Brain Res 1320, 130-134 (2010).

61. Roffman, J. L. et al. Genetic variation throughout the folate metabolic pathway influences negative symptom severity in schizophrenia. Schizophr. Bull. 39, 330-338 (2013).

62. El-Hadidy, M. A., Abdeen, H. M., Abd El-Aziz, S. M. \& Al-Harrass, M. MTHFR gene polymorphism and age of onset of schizophrenia and bipolar disorder. Biomed. Res Int 2014, 318483 (2014).

63. Peerbooms, O. L. et al. Meta-analysis of MTHFR gene variants in schizophrenia, bipolar disorder and unipolar depressive disorder: evidence for a common genetic vulnerability? Brain Behav. Immun. 25, 1530-1543 (2011).

64. Chen, Z. et al. C677T methylenetetrahydrofolate reductase gene polymorphisms in bipolar disorder: an association study in the Chinese population and a meta-analysis of genetic association studies. Neurosci. Lett. $\mathbf{4 4 9}$ 48-51 (2009).

65. Cohen-Woods, S. et al. The Bipolar Association Case-Control Study (BACCS) and meta-analysis: no association with the 5,10-Methylenetetrahydrofolate reductase gene and bipolar disorder. Am. J. Med Genet B Neuropsychiatr Genet 153B, 1298-1304 (2010).

66. Arzaghi, S. M., Shariat, A. H.-N. S. V., Ghodsipour, A., Shams, J. \& Larijani, B. C677T Methylenetetrahydrofolate Reductase (MTHFR) gene polymorphism in schizophrenia and bipolar disorder-an association study in Iranian population. Iran. J. Psychiatry 6, 1-6 (2011).

67. Kelly, C. B. M. A. P. \& Johnston, T. G. et al. The MTHFR C677T polymorphism is associated with depressive episodes in patients from Northern Ireland. J. Psychopharmacol. 18, 567 (2004).

68. Bousman, C. A. P. M. et al. Methylenetetrahydrofolate Reductase (MTHFR) genetic variation and major depressive disorder prognosis-a five-year prospective cohort study of primary care attendees. Am. J. Med. Genet. Part B Neuropsychiatr. Genet. 165, 68-76 (2013).

69. Folate, E. V. S. vitamin B12 and homocysteine, and the MTHFR 677C>T polymorphism in anxiety and depression. The Hordaland Homocysteine Study. Arch. Gen. Psychiatry 60, 618-626 (2003).

70. Słopien, R. J. K. et al. Polymorphic variants of genes encoding MTHFR, MTR, and MTHFD1 and the risk of depression in postmenopausal women in Poland. Maturitas 61, 252-255 (2008).

71. Lok, A. B. C. L. H. \& Koeter, M. W. J. et al. Interaction between the MTHFR C677T polymorphism and traumatic childhood events predicts depression. Transl. Psychiatry 3, e288 (2013).

72. Delport, D. et al. Significance of dietary folate intake, homocysteine levels and MTHFR 677 C>T genotyping in South African patients diagnosed with depression: test development for clinical application. Metab. Brain Dis. 29, 377-384 (2014).

73. Wu, Y. L. et al. Association between MTHFR C677T polymorphism and depression: an updated meta-analysis of 26 studies. Prog. Neuropsychopharmacol. Biol. Psychiatry 46, 78-85 (2013).

74. Gaysina, D. et al. No association with the 5,10-methylenetetrahydrofolate reductase gene and major depressive disorder: results of the depression case control (DeCC) study and a meta-analysis. Am. J. Med Genet B 147b, 699-706 (2008).

75. Shen, $X$. et al. Association analysis of COMT/MTHFR polymorphisms and major depressive disorder in Chinese Han population. J. Affect Disord. 161, 73-78 (2014).

76. Moorthy, D. et al. Status of vitamins B-12 and B-6 but not of folate, homocysteine, and the methylenetetrahydrofolate reductase C677T polymorphism are associated with impaired cognition and depression in adults. J. Nutr. 142, 1554-1560 (2012).

77. Lizer, M. H., Bogdan, R. L. \& Kidd, R. S. Comparison of the frequency of the methylenetetrahydrofolate reductase (MTHFR) C677T polymorphism in depressed versus nondepressed patients. J. Psychiatr. Pract. 17, 404-409 (2011).

78. Almeida, O. P. et al. Contribution of the MTHFR gene to the causal pathway for depression, anxiety and cognitive impairment in later life. Neurobiol. Aging 26, 251-257 (2005).

79. Bondy, B. et al. Combined action of the ACE D- and the G-protein beta3 Tallele in major depression: a possible link to cardiovascular disease? Mol. Psychiatry 7, 1120-1126 (2002).

80. Pasca, S. P. et al. One carbon metabolism disturbances and the C677T MTHFR gene polymorphism in children with autism spectrum disorders. J. Cell Mol. Med 13, 4229-4238 (2009).

81. Goin-Kochel, R. P. et al. The MTHFR 677C-->T polymorphism and behaviors in children with autism: exploratory genotype-phenotype correlations. Autism Res 2, 98-108 (2009).

82. Schmidt, R. J. et al. Prenatal vitamins, one-carbon metabolism gene variants, and risk for autism. Epidemiology 22, 476-485 (2011).

83. Guo, T., Chen, H., Liu, B., Ji, W. \& Yang, C. Methylenetetrahydrofolate reductase polymorphisms C677T and risk of autism in the Chinese Han population. Genet Test. Mol. Biomark. 16, 968-973 (2012).

84. Park, J. et al. MTHFR 1298A > C is a risk factor for autism spectrum disorder in the Korean population. Psychiatry Res 215, 258-259 (2014).

85. Krull, K. R. et al. Folate pathway genetic polymorphisms are related to attention disorders in childhood leukemia survivors. J. Pediatr. 152, 101-105 (2008).

86. Gokcen, C. K. N. \& Pekgor, A. Methylenetetrahydrofolate reductase gene polymorphisms in children with attention deficit hyperactivity disorder. Int. J. Med. Sci. 8, 523-528 (2011)

87. Ergul, E., Sazci, A. \& Kara, I. Methylenetetrahydrofolate reductase gene polymorphisms in Turkish children with attention-deficit/hyperactivity disorder. Genet Test. Mol. Biomark. 16, 67-69 (2012).

88. Engbersen, A. M. T. et al. Thermolabile 5,10-methylenetetrahydrofolate reductase as a cause of mild hyperhomocysteinemia. Am. J. Human. Genet 56, 142-150 (1995).

89. Yano, $\mathrm{H}$. et al. Mutations of the MTHFR gene $(428 \mathrm{C}>\mathrm{T}$ and $[458 \mathrm{G}>\mathrm{T}$ +459 C $>$ T]) markedly decrease MTHFR enzyme activity. Neurogenetics $\mathbf{5}$, 135-140 (2004).

90. Huang, L., Zhang, J., Hayakawa, T. \& Tsuge, H. Assays of methylenetetrahydrofolate reductase and methionine synthase activities by monitoring 5methyltetrahydrofolate and tetrahydrofolate using high-performance liquid chromatography with fluorescence detection. Anal. Biochem 299, 253-259 (2001).

91. Kauwell, G. P. et al. Methylenetetrahydrofolate reductase mutation (677C-- > T) negatively influences plasma homocysteine response to marginal folate intake in elderly women. Metabolism 49, 1440-1443 (2000).

92. Rampersaud, G. C. et al. Methylation decreases in response to moderate folate depletion in elderly women. Am. J. Clin. Nutr. 72, 998-1003 (2000).

93. Susser, E. et al. Schizophrenia after prenatal famine-further evidence. Arch. Gen. Psychiat 53, 25-31 (1996).

94. Goff, D. C. et al. Folate, homocysteine, and negative symptoms in schizophrenia. Am. J. Psychiatry 161, 1705-1708 (2004).

95. Godfrey, P. S. et al. Enhancement of recovery from psychiatric illness by methylfolate. Lancet 336, 392-395 (1990).

96. Picker, J. D. \& Coyle, J. T. Do maternal folate and homocysteine levels play a role in neurodevelopmental processes that increase risk for schizophrenia? Han. Rev. Psychiatry 13, 197-205 (2005).

97. Kontis, D. et al. COMT and MTHFR polymorphisms interaction on cognition in schizophrenia: an exploratory study. Neurosci. Lett. 537, 17-22 (2013).

98. AR, LewisS. J. et al. Folic acid supplementation during pregnancy may protect against depression 21 months after pregnancy, an effect modified by MTHFR C677T genotype. Eur. J. Clin. Nutr. 66, 97-103 (2012).

99. Schmidt, R. J. et al. Maternal periconceptional folic acid intake and risk of autism spectrum disorders and developmental delay in the CHARGE (CHildhood Autism Risks from Genetics and Environment) case-control study. Am. J. Clin. Nutr. 96, 80-89 (2012).

100. dos Santos, P. A. L. D. et al. MTHFR C677T is not a risk factor for autism spectrum disorders in South Brazil. Psychiatr. Genet. 20, 187-189 (2010).

101. Sener, E. F., Oztop, D. B. \& Ozkul, Y. MTHFR gene C677T polymorphism in autism spectrum disorders. Genet Res Int 2014, 698574 (2014).

102. Saha, T., \& Chatterjee, M. \& Sinha, S. \& Rajamma, U. \& Mukhopadhyay, K. Components of the folate metabolic pathway and ADHD core traits: an exploration in eastern Indian probands. J Hum Genet 62, 687-695 (2017). 